\title{
CONSTRUCTION OF BALL SPACES AND THE NOTION OF CONTINUITY
}

\author{
René Bartsch, Katarzyna Kuhlmann, and Franz-Viktor Kuhlmann \\ (Received 14 June, 2020)
}

\begin{abstract}
Spherically complete ball spaces provide a simple framework for the encoding of completeness properties of various spaces and ordered structures. This allows to prove generic versions of theorems that work with these completeness properties, such as fixed point theorems and related results. For the purpose of applying the generic theorems, it is important to have methods for the construction of new spherically complete ball spaces from existing ones. Given various ball spaces on the same underlying set, we discuss the construction of new ball spaces through set theoretic operations on the balls. A definition of continuity for functions on ball spaces leads to the notion of quotient spaces. Further, we show the existence of products and coproducts and use this to derive a topological category associated with ball spaces.
\end{abstract}

\section{Introduction}

Ball spaces have been introduced in 6 as a framework for the proof of generic fixed point theorems for functions which in some way are contracting. Since then, the development of their theory and their applications has led to several articles $(\mathbf{1}, \mathbf{2}, \mathbf{7}, \mathbf{4}, \mathbf{8}, \mathbf{5})$.

A ball space is a pair $(X, \mathcal{B})$ consisting of a nonempty set $X$ and a nonempty set of distinguished nonempty subsets $B$ of $X$. If we denote the power set of $X$ by $\mathcal{P}(X)$, this means that

$$
\emptyset \neq \mathcal{B} \subseteq \mathcal{P}(X) \backslash\{\emptyset\} .
$$

The elements $B$ of $\mathcal{B}$ will be called balls, in analogy to the case of metric (or ultrametric) balls. In analogy to the case of ultrametric spaces, we will call a nonempty collection $\mathcal{N}$ of balls in $\mathcal{B}$ a nest of balls (in $\mathcal{B}$ ) if it is totally ordered by inclusion. We will say that $(X, \mathcal{B})$ is spherically complete if the intersection $\bigcap \mathcal{N}$ of each nest $\mathcal{N}$ of balls in $\mathcal{B}$ is nonempty. The notions of "nest of balls" and "spherically complete" are taken over from the well developed theory of ultrametric spaces, valued abelian groups and valued fields, which also inspired the idea of ball spaces.

Ball spaces can be derived from various settings, such as metric spaces, ultrametric spaces, topological spaces (where it is convenient to take the balls to be the nonempty closed sets), partially ordered sets, lattices. The generic fixed point theorems that hold in spherically complete ball spaces then allow specializations

2010 Mathematics Subject Classification Primary 54A05; Secondary 54H25, 12J15, 03E20, 18 B05. Key words and phrases: fixed point theorems, ball spaces, products and coproducts, quotient spaces, continuity.

The third author is supported by Opus grant 2017/25/B/ST1/01815 from the National Science Centre of Poland. 
to all of these settings, in which spherically completeness is equivalent to natural completeness or compactness properties (cf. [1]). Let us mention at this point that when we associate to an ultrametric space the ball space consisting of all closed ultrametric balls, then this ball space is spherically complete if and only if the ultrametric space is (in fact, the notion of spherical completeness for ball spaces has been taken over from ultrametric spaces). Similarly, when we associate to a topological space the ball space consisting of all nonempty closed sets, then this ball space is spherically complete if and only if the topological space is compact.

The general framework of ball spaces allows transfers of concepts and approaches between these various settings (again, cf. [1]). The standard procedure for this is to first transfer a concept and associated results from one setting to that of ball spaces and then specialize from there to another setting. Let us give some examples.

Take a function $f$ from a ball space $(X, \mathcal{B})$ to a ball space $\left(X^{\prime}, \mathcal{B}^{\prime}\right)$. Under which conditions does $f$ transfer spherical completeness from one side to the other? This question has arisen in the setting where $X$ and $Y$ are ultrametric spaces and the aim was to prove coincidence theorems which for two given functions $f, g: X \rightarrow Y$ give conditions under which there exists $x \in X$ such that $f(x)=g(x)$. In [11] such theorems were proven (under the name "common point theorem") for the case of ultrametric spaces $X=X^{\prime}$. In 5 this task was then transferred to ball spaces and two types of generic coincidence theorems are proven: one where $(X, \mathcal{B})$ is spherically complete, and the other where $\left(X^{\prime}, \mathcal{B}^{\prime}\right)$ is spherically complete.

In order to answer the above question, it is a good idea to look at classical properties of functions. The notions of "continuous function" and "closed function" are commonly defined in metric, or more generally topological, spaces. Transferring these notions to ball spaces, we call $f: X \rightarrow X^{\prime}$ ball continuous if the preimage of every ball in $\mathcal{B}^{\prime}$ is a ball in $\mathcal{B}$, and ball closed if the image of every ball in $\mathcal{B}$ is a ball in $\mathcal{B}^{\prime}$. Then the following holds:

Theorem 1. If $f$ is ball continuous and $(X, \mathcal{B})$ is spherically complete, then so is $\left(X^{\prime}, \mathcal{B}^{\prime}\right)$. If $f$ is ball closed and finite-to-one, and if $\left(X^{\prime}, \mathcal{B}^{\prime}\right)$ is spherically complete, then so is $(X, \mathcal{B})$

These results are parts a) and e) of Theorem 5 in Section 3 , in which we study further conditions for the transfer of spherical completeness. It provides the necessary background for the definition of the notion of "quotient ball space" in the same section.

A second example for the transfer of notions is given by the notion of products. Inspired by the celebrated Tychonoff Theorem, we ask the question: is the product of spherically complete ball spaces again spherically complete (and in fact, how should the product be defined)? We will deal with these questions in Section 4 In [1] the ball spaces analogue of the Tychonoff Theorem is transferred to ultrametric spaces. The result that (correctly defined) products of spherically complete ultrametric spaces are again spherically complete has important applications in multidimensional versions of Hensel's Lemma as well as multidimensional and even infinite dimensional Implicit Function Theorems (see [3] ).

Having the notion of ball continuity at hand, we define in Section 4 the category of ball spaces, where the morphisms are the ball continuous functions. Our definition of products of ball spaces provides the products in this category. We also show 
the existence of coproducts and prove that coproducts of spherically complete ball spaces are again spherically complete (see Theorem 9).

In many situations the behaviour of ball spaces reminds us of topological spaces, and indeed, many inspiring examples come from a topological context. Hence in order to provide topological methods, we derive from the category of ball spaces the category of augmented ball spaces by adding the full space and the empty set to the sets of balls. We prove:

Theorem 2. The category of augmented ball spaces is topological.

For definitions and details, see Section 5 Note that this result implies that the category can be embedded in a cartesian closed topological category, ensuring that we have more than the weak (pointwise) function spaces at hand, which belong to the same kind of structure (see e.g. [13).

Apart from forming quotient spaces and products there are many more procedures to construct a new ball space $(X, \mathcal{B})$ from a given ball space or a set of ball spaces $\left(X_{i}, \mathcal{B}_{i}\right), i \in I$. We will discuss some of them in this paper. Since most of the generic fixed point theorems work for spherically complete ball spaces, it is an important question under which conditions the spaces $\left(X_{i}, \mathcal{B}_{i}\right)$ being spherically complete implies that so is $(X, \mathcal{B})$ (as is the case for products and coproducts). The simplest case for this to work is when we take the union of the sets of balls of two ball spaces on the same set $X$ (see Proposition 11). For certain other operations, however, we may need the ball spaces to satisfy a stronger form of spherical completeness; a hierarchy of them will be introduced in Section 2.1. This for instance happens when we take a single ball space $(X, \mathcal{B})$ and want to replace $\mathcal{B}$ by the set of all finite unions of balls in $\mathcal{B}$ (see Theorem 12). We will also study which of the stronger forms of spherical completeness are preserved under forming products, coproducts and unions of two ball spaces. For the study of further operations on the set of balls and the connection with topologies, see [1].

Returning to the case of several ball spaces on a given set $X$, we will discuss in Section 6.2 the particularly interesting natural example of ordered abelian groups and fields. For the case of fields, S. Shelah introduced in $[\mathbf{1 4}$ the notion of symmetrical completeness which means that for every Dedekind cut, the cofinality of the left cut set is different from the coinitiality of the right cut set (as is the case in the reals). He showed that arbitrarily large symmetrically complete ordered fields exist. With a different construction idea, the existence result is reproven in 8 and generalized to the case of ordered abelian groups and ordered sets. For the ordered sets, the existence result in fact follows already from the comprehensive work of Hausdorff. It is also shown in $\mathbf{8}$ that symmetrical completeness is equivalent to the spherical completeness of the induced order ball space, which is defined as follows. If $(I, \leq)$ is any nonempty totally ordered set, then we take $\mathcal{B}_{o}$ to consist of all closed bounded intervals $[a, b]$ with $a, b \in I, a \leq b$. We call $\left(I, \mathcal{B}_{o}\right)$ the order ball space associated with $(I, \leq)$.

On the other hand, non-archimedean ordered abelian groups and fields carry a nontrivial natural valuation (cf. Section 2.3), which induces an ultrametric ball space (cf. Section 2.2). This ball space is always spherically complete when the order ball space is, but the two spaces are distinct. It should be noted that the balls in the ultrametric ball space are precisely all cosets of the principal convex 
subgroups; here a convex subgroup is called principal if it is the smallest among all convex subgroups containing a fixed element. Note that in any ordered set, a subset $S$ is called convex if $a, b \in S$ and $a<c<b$ imply that $c \in S$.

We can apply Proposition 11 to these two ball spaces, but we cannot apply Theorem 12 as the order ball space and hence also the union of the two ball spaces does not satisfy the necessary requirements. However, in Section 6.2 we prove:

Theorem 3. Take a symmetrically complete ordered group or field $G$ and let $\mathcal{B}$ be the set of all convex sets in $G$ that are finite unions of closed bounded intervals and ultrametric balls. Then $(G, \mathcal{B})$ is spherically complete.

We also show that the convex sets appearing in the theorem can always be represented as two distinct ultrametric balls connected by a closed interval (hence we gave them the nickname "bar-bells").

Open question: Does the theorem also hold if the condition "convex" is removed?

\section{Preliminaries}

2.1. A hierarchy of ball spaces. In [1] we introduce and study the following hierarchy of spherical completeness properties:

$\mathbf{S}_{1}$ : The intersection of each nest in $(X, \mathcal{B})$ is nonempty.

$\mathbf{S}_{2}$ : The intersection of each nest in $(X, \mathcal{B})$ contains a ball.

$\mathbf{S}_{3}$ : The intersection of each nest in $(X, \mathcal{B})$ contains maximal balls.

$\mathbf{S}_{4}$ : The intersection of each nest in $(X, \mathcal{B})$ contains a largest ball.

$\mathbf{S}_{5}$ : The intersection of each nest in $(X, \mathcal{B})$ is a ball.

$\mathbf{S}_{i}^{c}$ : The same as $\mathbf{S}_{i}$, but with "centered system" in place of "nest".

Here, a centered system of balls is a collection of balls such that the intersection of any finite number of balls in the collection is nonempty.

2.2. Ball spaces associated with ultrametric spaces and valuations. An ultrametric space is a pair $(X, u)$ where $X$ is a set and $u: X \times X \rightarrow \Gamma$ with $\Gamma$ a totally ordered set with largest element $\infty$, such that:

(UM1) $u(x, y)=\infty$ if and only if $x=y$;

(UM2) $u(x, y)=u(y, x)$;

(UM3) $u(x, z) \geq \min \{u(x, y), u(y, z)\}$.

(UM3) is called the ultrametric triangle law. The image

$$
u X:=u(X \times X) \backslash\{\infty\}
$$

is called the value set of $u$. A generalization of the notion of ultrametric space works with partially ordered value sets $u X$ in place of totally ordered ones (see 10, 12, 1, 2]), but we will not need this generalization in the present paper.

One frequent source of ultrametrics are valuations: if $v$ is a valuation on a field or an abelian group, then one may define $u(a, b):=v(a-b)$. With this definition, the above axioms are satisfied if $v$ has values in $\Gamma \cup\{\infty\}$ and is written as a Krull valuation, that is, it satisfies the following axioms:

(V1) $v x=\infty$ if and only if $x=0$;

(V2) $v(x-y) \geq \min \{v x, v y\} \quad$ (ultrametric triangle law). 
Note that for valuations on fields we require in addition that $\Gamma$ is an ordered abelian group together with $\infty$ (an element larger than all elements of the group) and the following axiom holds:

(VH) $v(x y)=v x+v y \quad$ (homomorphism law).

Take an ultrametric space $(X, u)$. For $x \in X$ and $\gamma \in u X \cup\{\infty\}$, the set

$$
B_{\gamma}(x):=\{x \in X \mid u(x, y) \geq \gamma\}
$$

is called the closed ball of radius $\gamma$ around $x$. Further, we define

$$
B(x, y):=B_{u(x, y)}(x)=B_{u(x, y)}(y),
$$

where the latter equality follows from the fact that in an ultrametric space every element of a ball is a center. If $X$ is nonempty, then $\left(X, \mathcal{B}_{u}\right)$ with

$$
\mathcal{B}_{u}:=\{B(x, y) \mid x, y \in X\}
$$

is a ball space, which we call the ultrametric ball space.

From the ultrametric triangle law it follows that any two balls in $\mathcal{B}_{u}$ are already comparable by inclusion once they have a nonempty intersection. It follows that every centered system of balls in $\mathcal{B}_{u}$ is in fact a nest of balls.

2.3. Nonarchimedean orderings and the natural valuation. Take an ordered abelian group $(G, \leq)$. Two elements $a, b \in G$ are called archimedean equivalent if there is some $n \in \mathbb{N}$ such that $n|a| \geq|b|$ and $n|b| \geq|a|$. The ordered abelian group $(G, \leq)$ is archimedean ordered if all nonzero elements are archimedean equivalent. If $0 \leq a<b$ and $n a<b$ for all $n \in \mathbb{N}$, then we say that " $a$ is infinitesimally smaller than $b$ ". We denote by $v a$ the archimedean equivalence class of $a$. The set of archimedean equivalence classes can be ordered by setting $v a>v b$ if and only if $|a|<|b|$ and $a$ and $b$ are not archimedean equivalent, that is, if $n|a|<|b|$ for all $n \in \mathbb{N}$. We write $\infty:=v 0$; this is the maximal element in the linearly ordered set of equivalence classes. The function $a \mapsto v a$ is a group valuation on $G$, i.e., it satisfies (V1) and (V2). By definition,

$$
0 \leq a \leq b \Longrightarrow v a \geq v b .
$$

The set $v G:=\{v g \mid 0 \neq g \in G\}$ is called the value set of the valued abelian group $(G, v)$.

If $(K, \leq)$ is an ordered field, then we consider the natural valuation on its ordered additive group and define $v a+v b:=v(a b)$. This turns the set of archimedean classes into an ordered abelian group, with neutral element $0:=v 1$ and inverses $-v a=v\left(a^{-1}\right)$. In this way, $v$ becomes a field valuation.

As shown in Section 2.2 above, the natural valuation of an ordered abelian group or ordered field induces an ultrametric ball space $\mathcal{B}_{u}$. 


\section{Ball Continuity and Quotient Ball Spaces}

Take two ball spaces $(X, \mathcal{B})$ and $\left(X^{\prime}, \mathcal{B}^{\prime}\right)$ and a function $f: X \rightarrow X^{\prime}$. We will call $f$ ball continuous if the preimage of every ball in $\mathcal{B}^{\prime}$ is a ball in $\mathcal{B}$, that is,

$$
\left\{f^{-1}\left(B^{\prime}\right) \mid B^{\prime} \in \mathcal{B}^{\prime}\right\} \subseteq \mathcal{B} .
$$

We will call $f$ ball closed if the image of every ball in $\mathcal{B}$ is a ball in $\mathcal{B}^{\prime}$.

Lemma 4. Take three ball spaces $\left(X, \mathcal{B}_{X}\right),\left(Y, \mathcal{B}_{Y}\right)$ and $\left(Z, \mathcal{B}_{Z}\right)$, and functions $f: X \rightarrow Y$ and $g: Y \rightarrow Z$. Then $g \circ f$ is ball continuous if $f$ and $g$ are. Likewise, $g \circ f$ is ball closed if $f$ and $g$ are.

Proof. For the first assertion, note that for every $B \in \mathcal{B}_{Z},(g \circ f)^{-1}(B)=$ $f^{-1}\left(g^{-1}(B)\right)$. The second assertion is obvious.

The next theorem gives conditions for functions to preserve spherical completeness in one or the other ditrection.

Theorem 5. Take two ball spaces $(X, \mathcal{B})$ and $\left(X^{\prime}, \mathcal{B}^{\prime}\right)$, and a function $f: X \rightarrow X^{\prime}$.

a) If $f$ is ball continuous and $(X, \mathcal{B})$ is spherically complete, then so is $\left(X^{\prime}, \mathcal{B}^{\prime}\right)$.

b) Assume that $f$ is surjective and

$$
\mathcal{B} \subseteq\left\{f^{-1}\left(B^{\prime}\right) \mid B^{\prime} \in \mathcal{B}^{\prime}\right\} .
$$

If $\left(X^{\prime}, \mathcal{B}^{\prime}\right)$ is spherically complete, then so is $(X, \mathcal{B})$.

c) Assume that $f$ is surjective or $\left(X^{\prime}, \mathcal{B}^{\prime}\right)$ is an $\mathbf{S}_{2}$ ball space, and that

$$
\mathcal{B}=\left\{f^{-1}\left(B^{\prime}\right) \mid B^{\prime} \in \mathcal{B}^{\prime}\right\} .
$$

Then $(X, \mathcal{B})$ is spherically complete if and only if $\left(X^{\prime}, \mathcal{B}^{\prime}\right)$ is.

d) If (3) holds and $f$ is surjective, then

$$
\mathcal{B}^{\prime}=\{f(B) \mid B \in \mathcal{B}\}
$$

and $f$ induces an isomorphism of the partially ordered sets $\mathcal{B}$ and $\mathcal{B}^{\prime}$.

e) $f: X \rightarrow X^{\prime}$ is ball closed and finite-to-one, and if $\left(X^{\prime}, \mathcal{B}^{\prime}\right)$ is spherically complete, then so is $(X, \mathcal{B})$.

Proof. a) Take a nest $\mathcal{N}^{\prime}=\left(B_{i}^{\prime}\right)_{i \in I}$ of balls in $\left(X^{\prime}, \mathcal{B}^{\prime}\right)$. Set $\mathcal{N}=\left(f^{-1}\left(B_{i}^{\prime}\right)\right)_{i \in I}$. By assumption, we have that $f^{-1}\left(B_{i}^{\prime}\right) \in \mathcal{B}$ for all $i \in I$, For any $i, j \in I$ we have that $B_{i}^{\prime} \subseteq B_{j}^{\prime}$ or $B_{j}^{\prime} \subseteq B_{i}^{\prime}$, hence also $f^{-1}\left(B_{i}^{\prime}\right) \subseteq f^{-1}\left(B_{j}^{\prime}\right)$ or $f^{-1}\left(B_{j}^{\prime}\right) \subseteq$ $f^{-1}\left(B_{i}^{\prime}\right)$. This proves that $\mathcal{N}$ is a nest in $\mathcal{B}$. As $(X, \mathcal{B})$ is spherically complete, $\bigcap \mathcal{N}$ is nonempty. Since $f(\bigcap \mathcal{N}) \subseteq B_{i}^{\prime}$ for all $i \in I$, it follows that $f(\bigcap \mathcal{N}) \subseteq$ $\bigcap \mathcal{N}^{\prime}$, which shows that the latter is nonempty.

b) Take a nest $\mathcal{N}=\left(B_{i}\right)_{i \in I}$ of balls in $(X, \mathcal{B})$. Set $\mathcal{N}^{\prime}=\left(f\left(B_{i}\right)\right)_{i \in I}$. By assumption, we have that every $B_{i}$ is the preimage of a ball $B_{i}^{\prime}$ in $\mathcal{B}^{\prime}$, hence $B_{i}=f^{-1}\left(B_{i}^{\prime}\right)$ for all $i \in I$. For any $i, j \in I$ we have that $B_{i} \subseteq B_{j}$ or $B_{j} \subseteq B_{i}$, hence also $f\left(B_{i}\right) \subseteq f\left(B_{j}\right)$ or $f\left(B_{j}\right) \subseteq f\left(B_{i}\right)$. This proves that $\mathcal{N}^{\prime}$ is a nest in $\mathcal{B}^{\prime}$. As $\left(X^{\prime}, \mathcal{B}^{\prime}\right)$ is spherically complete, $\cap \mathcal{N}^{\prime}$ is nonempty. Take $x^{\prime} \in \bigcap \mathcal{N}^{\prime}$. Then $x^{\prime} \in B_{i}^{\prime}$ for all $i \in I$. Pick some preimage $x \in X$ of $x^{\prime}$ under $f$; this is possible since $f$ is assumed surjective. Then $x \in f^{-1}\left(B_{i}^{\prime}\right)=B_{i}$ for all $i \in I$. Hence $x \in \bigcap \mathcal{N}$, showing that this intersection is nonempty. 
c) If $f$ is surjective, everything follows from assertions a) and b). If $f$ is not surjective, but $\left(X^{\prime}, \mathcal{B}^{\prime}\right)$ is an $\mathbf{S}_{2}$ ball space, then we have to modify the previous proof in order to show that some $x^{\prime} \in \bigcap \mathcal{N}^{\prime}$ has a preimage in $X$. Since $\left(X^{\prime}, \mathcal{B}^{\prime}\right)$ is $\mathbf{S}_{2}, \cap \mathcal{N}^{\prime}$ contains a ball $B^{\prime}$. By $(3), f^{-1}\left(B^{\prime}\right)$ is a ball in $\mathcal{B}$ and hence nonempty. So there is an $x^{\prime} \in \bigcap \mathcal{N}^{\prime}$ which has a preimage in $X$.

d) A surjective function $f: X \rightarrow X^{\prime}$ induces an inclusion preserving bijection between all subsets of $X^{\prime}$ and their preimages. Condition (3) implies (4) and that the restriction of $f$ to $\mathcal{B}^{\prime}$ is a bijection between $\mathcal{B}^{\prime}$ and $\mathcal{B}$.

e) Take a nest $\mathcal{N}=\left(B_{i}\right)_{i \in I}$ of balls in $(X, \mathcal{B})$. Set $\mathcal{N}^{\prime}=\left(f\left(B_{i}\right)\right)_{i \in I}$. By assumption, each $f\left(B_{i}\right)$ is a ball in $\mathcal{B}^{\prime}$, and since $f$ preserves inclusion between balls, $\mathcal{N}^{\prime}$ is a nest. As $\left(X^{\prime}, \mathcal{B}^{\prime}\right)$ is spherically complete, $\cap \mathcal{N}^{\prime}$ is nonempty. Take $x^{\prime} \in \bigcap \mathcal{N}^{\prime}$. Then $x^{\prime} \in f\left(B_{i}\right)$ for all $i \in I$. Among the finitely many preimages of $x^{\prime}$ under $f$ there must be at least one that is contained in all $B_{i}$. This element then lies in $\bigcap \mathcal{N}$, showing that the intersection is nonempty.

Take any function $f: X \rightarrow X^{\prime}$. We define functions

$$
\varphi_{f}: \mathcal{P}(X) \rightarrow \mathcal{P}\left(X^{\prime}\right) \text { and } \psi_{f}: \mathcal{P}\left(X^{\prime}\right) \rightarrow \mathcal{P}(X)
$$

by setting $\varphi_{f}(S):=f(S)$ for each set $S \subseteq X$ and $\psi_{f}\left(S^{\prime}\right):=f^{-1}\left(S^{\prime}\right)$ for each set $S^{\prime} \subseteq X^{\prime}$. If $\mathcal{S} \subseteq \mathcal{P}(X)$ and $\mathcal{S}^{\prime} \subseteq \mathcal{P}\left(X^{\prime}\right)$, then in accordance with our general use for functions, $\varphi_{f}(\mathcal{S})=\left\{\varphi_{f}(S) \mid S \in \mathcal{S}\right\}$ and $\psi_{f}\left(\mathcal{S}^{\prime}\right)=\left\{\psi_{f}\left(S^{\prime}\right) \mid S^{\prime} \in \mathcal{S}^{\prime}\right\}$. If $(X, \mathcal{B})$ is a ball space, then $\mathcal{B}$ is just a nonempty subset of $\mathcal{P}(X) \backslash\{\emptyset\}$. We observe that then $\varphi_{f}(\mathcal{B})$ is a nonempty subset of $\mathcal{P}\left(X^{\prime}\right) \backslash\{\emptyset\}$. Similarly, if $f$ is surjective, then $\emptyset \neq \psi_{f}\left(\mathcal{B}^{\prime}\right) \subseteq \mathcal{P}(X) \backslash\{\emptyset\}$.

Every nest of balls in $\mathcal{B}$ is also a ball space on $X$, but it has the special property of being totally ordered by inclusion. So we are interested in the question when this property is preserved by $\varphi_{f}$ and $\psi_{f}$. The following is a corollary to the previous proof.

Corollary 6. Take two ball spaces $(X, \mathcal{B})$ and $\left(X^{\prime}, \mathcal{B}^{\prime}\right)$ and a function $f: X \rightarrow X^{\prime}$.

a) If $f$ is ball continuous and $\mathcal{N}^{\prime}$ is a nest of balls in $\mathcal{B}^{\prime}$, then $\psi_{f}\left(\mathcal{N}^{\prime}\right)$ is a nest of balls in $\mathcal{B}$.

b) If $f$ is ball closed and $\mathcal{N}$ is a nest of balls in $\mathcal{B}$, then $\varphi_{f}(\mathcal{N})$ is a nest of balls in $\mathcal{B}^{\prime}$.

Take two ball spaces $(X, \mathcal{B})$ and $\left(X^{\prime}, \mathcal{B}^{\prime}\right)$. If there is a surjective function $f$ : $X \rightarrow X^{\prime}$ such that (3) holds, then we call $\left(X^{\prime}, \mathcal{B}^{\prime}\right)$ a quotient ball space of $(X, \mathcal{B})$ (induced by the function $f$ ). Note that $\mathcal{B}$ is the coarsest of all ball spaces $\mathcal{S}$ on $X$ such that $f$ is a ball continuous function from $(X, \mathcal{S})$ to $\left(X^{\prime}, \mathcal{B}^{\prime}\right)$, and that $\mathcal{B}^{\prime}$ is the finest of all ball spaces $\mathcal{S}^{\prime}$ on $X^{\prime}$ such that $f$ is a ball continuous function from $(X, \mathcal{B})$ to $\left(X^{\prime}, \mathcal{S}^{\prime}\right)$.

\section{Products and Coproducts}

We define the category of ball spaces to consist of all ball spaces as objects and the ball continuous functions between them as morphisms. In this section we show that products and coproducts exist in this category.

Theorem 7. The category of ball spaces admits products and coproducts. 
For the proof of the theorem, we will explicitly construct these objects. Take ball spaces $\left(Y_{i}, \mathcal{B}_{i}\right), i \in I$.

1) Products. We set $X=\prod_{i \in I} Y_{i}$ and denote by $p_{i}: X \rightarrow Y_{i}$ the projection from $X$ to $Y_{i}$. We set

$$
\mathcal{B}:=\left\{\prod_{i \in I} B_{i} \mid \text { for some } k \in I, B_{k} \in \mathcal{B}_{k} \text { and } \forall i \neq k: B_{i}=Y_{i}\right\} .
$$

Since the sets $\mathcal{B}_{i}$ are nonempty, it follows that $\mathcal{B} \neq \emptyset$, and as no ball in any $\mathcal{B}_{i}$ is empty, it follows that no member of $\mathcal{B}$ is empty. Hence $(X, \mathcal{B})$ is a ball space.

The definition of $\mathcal{B}$ yields that all projections are ball continuous. If $Y_{i} \in \mathcal{B}_{i}$ for all $i \in I$, then they are also ball closed.

In order to prove that our definition yields products in the category of ball spaces, we have to show that for any ball space $\left(Z, \mathcal{B}_{Z}\right)$ and ball continuous functions $f_{i}: Z \rightarrow Y_{i}$ there is a unique ball continuous function $g: Z \rightarrow X$ such that $p_{i} \circ g=f_{i}$ for all $i \in I$. The latter forces $g(z)=\left(f_{i}(z)\right)_{i \in I}$ for all $z \in Z$; this ensures uniqueness. It remains to show that $g$ is ball continuous. A ball $B \in \mathcal{B}$ is of the form $\prod_{i \in I} B_{i}$ as in 5 . Then $g^{-1}(B)=f_{k}^{-1}\left(B_{k}\right)$, which is a ball in $\mathcal{B}_{Z}$ since $f_{k}$ is ball continuous. This shows that $g$ is ball continuous.

2) Coproducts. Take ball spaces $\left(Y_{i}, \mathcal{B}_{i}\right)$, let $X$ be the disjoint union $\dot{\bigcup}_{i \in I} Y_{i}$ and denote by $\iota_{i}: Y_{i} \rightarrow X$ the canonical embedding of $Y_{i}$ in $X$. We set

$$
\mathcal{B}:=\left\{\bigcup_{i \in I} \iota_{i}\left(B_{i}\right) \mid \forall i \in I: B_{i} \in \mathcal{B}_{i}\right\} .
$$

For the same reasons as before, $\mathcal{B} \neq \emptyset$ and no member of $\mathcal{B}$ is empty, hence again, $(X, \mathcal{B})$ is a ball space.

For all $j \in I$ we have that $\iota_{j}^{-1}\left(\bigcup_{i \in I} \iota_{i}\left(B_{i}\right)\right)=B_{j}$, so each $\iota_{j}$ is ball continuous. We have to show that for any ball space $\left(Z, \mathcal{B}_{Z}\right)$ and ball continuous functions $f_{i}: Y_{i} \rightarrow Z$ there is a unique ball continuous function $g: X \rightarrow Z$ such that $g \circ \iota_{i}=f_{i}$ for all $i \in I$. The latter forces $g(x)=f_{i}(y)$ when $y \in Y_{i}$ with $x=\iota_{i}(y)$; this ensures uniqueness. It remains to show that $g$ is ball continuous. Take a ball $B \in \mathcal{B}_{Z}$. Then $g^{-1}(B)=\bigcup_{i \in I} \iota_{i}\left(f_{i}^{-1}(B)\right)$. This is a ball in $\mathcal{B}$ because $f_{i}^{-1}(B) \in \mathcal{B}_{i}$ for all $i \in I$ as all $f_{i}$ are ball continuous.

Notation: We will denote the product defined above by $\prod_{i \in I}\left(Y_{i}, \mathcal{B}_{i}\right)$, and the coproduct by $\coprod_{i \in I}\left(Y_{i}, \mathcal{B}_{i}\right)$. Further, we may rewrite $\bigcup_{i \in I} \iota_{i}\left(B_{i}\right)$ as $\bigcup_{i \in I} B_{i}$.

Theorem 8. Take ball spaces $\left(Y_{i}, \mathcal{B}_{i}\right), i \in I$. Then the following assertions hold:

a) $\prod_{i \in I}\left(Y_{i}, \mathcal{B}_{i}\right)$ is spherically complete if and only if all ball spaces $\left(Y_{i}, \mathcal{B}_{i}\right), i \in I$, are spherically complete.

b) If at least one of the ball spaces $\left(Y_{i}, \mathcal{B}_{i}\right), i \in I$, is spherically complete, then $\coprod_{i \in I}\left(Y_{i}, \mathcal{B}_{i}\right)$ is spherically complete.

c) The following are equivalent:

i) all ball spaces $\left(Y_{i}, \mathcal{B}_{i}\right), i \in I$, are $\mathbf{S}_{2}$,

ii) $\prod_{i \in I}\left(Y_{i}, \mathcal{B}_{i}\right)$ is $\mathbf{S}_{2}$,

iii) $\coprod_{i \in I}\left(Y_{i}, \mathcal{B}_{i}\right)$ is $\mathbf{S}_{2}$.

The same holds with $\mathbf{S}_{3}$, with $\mathbf{S}_{4}$ and with $\mathbf{S}_{5}$ in place of $\mathbf{S}_{2}$. 
Proof. Let us first observe the following. If $I$ and $J$ are some index sets and $B_{i, j}$, $i \in I, j \in J$ are arbitrary sets, then

$$
\bigcap_{j \in J} \prod_{i \in I} B_{i, j}=\prod_{i \in I} \bigcap_{j \in J} B_{i, j} \quad \text { and } \bigcap_{j \in J} \bigcup_{i \in I} B_{i, j}=\bigcup_{i \in I} \bigcap_{j \in J} B_{i, j} .
$$

a) First assume that all ball spaces $\left(Y_{i}, \mathcal{B}_{i}\right), i \in I$, are spherically complete. Take a nest $\left(\prod_{i \in I} B_{i, j}\right)_{j \in J}$ in $\prod_{i \in I}\left(Y_{i}, \mathcal{B}_{i}\right)$. Then there is some $k \in I$ such that $B_{i, j}=Y_{i}$ for all $i \in I$ with $i \neq k$ and all $j \in J$, and $\left(B_{k, j}\right)_{j \in J}$ is a nest of balls in $\mathcal{B}_{k}$. Since $\left(Y_{k}, \mathcal{B}_{k}\right)$ is spherically complete, $\bigcap_{j \in J} B_{k, j} \neq \emptyset$. As $\bigcap_{j \in J} B_{i, j}=Y_{i}$ for $i \neq k$, we find that $\bigcap_{j \in J} \prod_{i \in I} B_{i, j}=\prod_{i \in I} \bigcap_{j \in J} B_{i, j} \neq \emptyset$. This proves that $\prod_{i \in I}\left(Y_{i}, \mathcal{B}_{i}\right)$ is spherically complete.

Now assume that $\prod_{i \in I}\left(Y_{i}, \mathcal{B}_{i}\right)$ is spherically complete. We have already seen that all projections are ball continuous. Hence it follows from part a) of Theorem 5 that for every $i \in I,\left(Y_{i}, \mathcal{B}_{i}\right)$ is spherically complete.

b) Assume that at least one of the ball spaces $\left(Y_{i}, \mathcal{B}_{i}\right), i \in I$, is spherically complete. Take a nest $\left(\dot{\bigcup}_{i \in I} B_{i, j}\right)_{j \in J}$ in $\coprod_{i \in I}\left(Y_{i}, \mathcal{B}_{i}\right)$. Since the unions are disjoint, $\dot{\bigcup}_{i \in I} B_{i, j_{1}} \subseteq \dot{\bigcup}_{i \in I} B_{i, j_{2}}$ implies that $B_{i, j_{1}} \subseteq B_{i, j_{2}}$ for all $i \in I$. This shows that for each $i \in I,\left(B_{i, j}\right)_{j \in J}$ is a nest in $\mathcal{B}_{i}$. If $\left(Y_{k}, \mathcal{B}_{k}\right)$ is spherically complete, then

$$
\emptyset \neq \bigcap_{j \in J} B_{k, j} \subseteq \bigcup_{i \in I} \bigcap_{j \in J} B_{i, j}=\bigcap_{j \in J} \bigcup_{i \in I} B_{i, j} .
$$

c) We keep the notations of the proofs of a) and b). If all ball spaces $\left(Y_{i}, \mathcal{B}_{i}\right)$, $i \in I$, are $\mathbf{S}_{2}$, then for each $i \in I, \bigcap_{j \in J} B_{i, j}$ contains a ball $B_{i}^{\prime}$. As in the proof of part a), we know that there is some $k \in I$ such that $B_{i, j}=Y_{i}$ for all $i \in I$ with $i \neq k$ and all $j \in J$. Thus we can take $B_{i}^{\prime}=Y_{i}$ for all $i \in I$ with $i \neq k$. Then $\bigcap_{j \in J} \prod_{i \in I} B_{i, j}=\prod_{i \in I} \bigcap_{j \in J} B_{i, j}$ contains the ball $\prod_{i \in I} B_{i}^{\prime}$. If all spaces are even $\mathbf{S}_{3}$ (or $\mathbf{S}_{4}$ ), then $B_{k}^{\prime}$ can be taken as maximal (or largest, respectively) ball in $\mathcal{B}_{k} \cup\left\{Y_{k}\right\}$ contained in $\bigcap_{j \in J} B_{k, j}$ and it follows that $\prod_{i \in I} B_{i}^{\prime}$ is a maximal (or largest, respectively) ball contained in $\prod_{i \in I} \bigcap_{j \in J} B_{i, j}$. If all spaces are $\mathbf{S}_{5}$, then $\bigcap_{j \in J} B_{k, j}$ is a ball in $\mathcal{B}_{k} \cup\left\{Y_{k}\right\}$ and hence $\bigcap_{j \in J} \prod_{i \in I} B_{i, j}=\prod_{i \in I} \bigcap_{j \in J} B_{i, j}$ is a ball. This proves that i) implies ii) in all four cases.

In view of the equality $\bigcap_{j \in J} \dot{\bigcup}_{i \in I} B_{i, j}=\dot{\bigcup}_{i \in I} \bigcap_{j \in J} B_{i, j}$ the above arguments can be readily adapted to prove that i) implies iii) in all three cases.

Now assume that $\prod_{i \in I}\left(Y_{i}, \mathcal{B}_{i}\right)$ is $\mathbf{S}_{2}$. Take $k \in I$ and a nest of balls $\mathcal{N}=$ $\left(B_{j}\right)_{j \in J}$ in $\left(Y_{k}, \mathcal{B}_{k}\right)$. We define the nest $\prod_{i \in I}\left(Y_{i}, \mathcal{B}_{i}\right)$ as in the proof of part a). By assumption, the intersection $\bigcap_{j \in J} \prod_{i \in I} B_{i, j}=\prod_{i \in I} \bigcap_{j \in J} B_{i, j}$ contains a ball $\prod_{i \in I} B_{i}^{\prime}$. It follows that $B_{k}^{\prime}$ is a ball in $\mathcal{B}_{k}$ that is contained in $\bigcap_{j \in J} B_{k, j}$. If $\prod_{i \in I}\left(Y_{i}, \mathcal{B}_{i}\right)$ is even $\mathbf{S}_{3}$ (or $\mathbf{S}_{4}$ ), then $\prod_{i \in I} B_{i}^{\prime}$ can be assumed to be a maximal (or largest, respectively) ball contained in $\bigcap_{j \in J} \prod_{i \in I} B_{i, j}$, which implies that $B_{k}^{\prime}$ is a maximal (or largest, respectively) ball contained in $\bigcap_{j \in J} B_{k, j}$. If $\prod_{i \in I}\left(Y_{i}, \mathcal{B}_{i}\right)$ is $\mathbf{S}_{5}$, then $\bigcap_{j \in J} \prod_{i \in I} B_{i, j}$ is a ball, which implies that $\bigcap_{j \in J} B_{k, j}$ is a ball in $\mathcal{B}_{k}$. We have proved that ii) implies i) in all three cases.

Replacing " $\prod_{i \in I}$ " by "Uं $\dot{U}_{i \in I}$ " gives that iii) implies i) in all four cases. 
At first glance it may be surprising that for $\coprod_{i \in I}\left(Y_{i}, \mathcal{B}_{i}\right)$ to be spherically complete it suffices that just one of the ball spaces $\left(Y_{i}, \mathcal{B}_{i}\right)$ is spherically complete, while for $\coprod_{i \in I}\left(Y_{i}, \mathcal{B}_{i}\right)$ to be $\mathbf{S}_{2}, \mathbf{S}_{3}, \mathbf{S}_{4}$ or $\mathbf{S}_{5}$, all ball spaces $\left(Y_{i}, \mathcal{B}_{i}\right)$ must have the same property. This is because for $\bigcap_{j \in J} \dot{\bigcup}_{i \in I} B_{i, j}$ to be nonempty it suffices that $\bigcap_{j \in J} B_{i, j}$ is nonempty for at least one $i \in I$, whereas for it to contain a ball, all $\bigcap_{j \in J} B_{i, j}$ must contain a ball.

From the previous theorem we immediately obtain the following result.

Theorem 9. The categories of spherically complete ball spaces, of $\mathbf{S}_{2}$ ball spaces, of $\mathbf{S}_{3}$ ball spaces, of $\mathbf{S}_{4}$ ball spaces and of $\mathbf{S}_{5}$ ball spaces, all of them with ball continuous functions as their morphisms, admit products and coproducts.

Let us conclude this section by giving an example which shows that the converse in part b) of Theorem 8 is in general not true. (However, it can be shown to be true when all $\mathcal{B}_{i}$ are countable.)

Example 10. Take $X_{1}=\omega, \mathcal{B}_{1}$ to be the set of all final segments in $\omega, X_{2}=\omega_{1}$, and $\mathcal{B}_{2}$ to be the set of all final segments in $\omega_{1}$. Then neither $\left(X_{1}, \mathcal{B}_{1}\right)$ nor $\left(X_{2}, \mathcal{B}_{2}\right)$ is spherically complete as in both cases, the intersection over all final segments is empty. Note, however, that the intersection over every countable nest of balls in $\left(X_{2}, \mathcal{B}_{2}\right)$ is nonempty, and thus the same is true in the coproduct of the two ball spaces. On the other hand, if $\mathcal{N}=\left(\dot{\bigcup}_{i \in\{1,2\}} B_{i, j}\right)_{j \in J}$ is an uncountable nest in $\coprod_{i \in\{1,2\}}\left(Y_{i}, \mathcal{B}_{i}\right)$, then we may w.l.o.g. assume that $J=\omega_{1}$ and that $j_{1}<j_{2}$ implies $B_{1, j_{2}} \subseteq B_{1, j_{1}}$ and $B_{2, j_{2}} \subseteq B_{2, j_{1}}$. Since $\mathcal{B}_{1}$ is countable, the balls $B_{1, j}$ must eventually become stationary, i.e., equal to one and the same ball $B_{1} \in \mathcal{B}_{1}$, which is then contained in the intersection of the nest $\mathcal{N}$.

\section{The Topological Category of Augmented Ball Spaces}

If $(X, \mathcal{B})$ is a ball space and $\mathcal{A}=\mathcal{B} \cup\{\emptyset, X\}$, or if $X$ is an arbitrary (not necessarily nonempty) set and $\mathcal{A}=\{\emptyset, X\}$, then we call $(X, \mathcal{A})$ an augmented ball space. Take two ball spaces $(X, \mathcal{B})$ and $\left(X^{\prime}, \mathcal{B}^{\prime}\right)$ and a ball continuous function $f: X \rightarrow X^{\prime}$. Since $f^{-1}(\emptyset)=\emptyset$ and $f^{-1}\left(X^{\prime}\right)=X, f$ also satisfies

$$
\left\{f^{-1}\left(A^{\prime}\right) \mid A^{\prime} \in \mathcal{A}^{\prime}\right\} \subseteq \mathcal{A},
$$

where $\mathcal{A}^{\prime}=\mathcal{B}^{\prime} \cup\left\{\emptyset, X^{\prime}\right\}$. Therefore we will also call $f$ a ball continuous function from $(X, \mathcal{A})$ to $\left(X^{\prime}, \mathcal{A}^{\prime}\right)$. Note that $f$ is always ball continuous when $\mathcal{A}^{\prime}=\{\emptyset, X\}$.

We define the category of augmented ball spaces to consist of all augmented ball spaces as objects, with the ball continuous functions between them as morphisms.

A category $\mathcal{C}$ is called topological if

(1) For all sets $X$ and all families $\left(f_{i},\left(X_{i}, \xi_{i}\right)\right)_{i \in I}$, indexed by a class $I$, of $\mathcal{C}$-objects $\left(X_{i}, \xi_{i}\right)$ and functions $f_{i}: X \rightarrow X_{i}$ there exists a unique initial $\mathcal{C}$-object $(X, \xi)$ on the set $X$, i.e., an object $(X, \xi)$ s.t. for all $\mathcal{C}$-objects $(Y, \eta)$ and maps $g: Y \rightarrow X$ the following holds:

$$
\begin{gathered}
g \in[(Y, \eta),(X, \xi)]_{\mathcal{C}} \Leftrightarrow \forall i \in I: f_{i} \circ g \in\left[(Y, \eta),\left(X_{i}, \xi_{i}\right)\right]_{\mathcal{C}} \\
(Y, \eta) \stackrel{g}{\longrightarrow}(X, \xi) \stackrel{f_{i}}{\longrightarrow}\left(X_{i}, \xi_{i}\right)
\end{gathered}
$$

That is: arbitrary initial structures exist. 
(2) Fibre-smallness: For all sets $X$, the class of $\mathcal{C}$-objects on $X$ is a set.

(3) On sets with at most one element exists exactly one $\mathcal{C}$-structure.

\section{Proof of Theorem 2 ,}

(1) The category admits initial objects. Take augmented ball spaces $\left(Y_{i}, \mathcal{A}_{i}\right)$, a set $X$, and functions $f_{i}: X \rightarrow Y_{i}, i \in I$. We set

$$
\mathcal{A}:=\left\{f_{i}^{-1}\left(A_{i}\right) \mid i \in I, A_{i} \in \mathcal{A}_{i}\right\} .
$$

Observe that $\emptyset=f_{i}^{-1}(\emptyset) \in \mathcal{A}$ and $X=f_{i}^{-1}\left(Y_{i}\right) \in \mathcal{A}$ since $\left(Y_{i}, \mathcal{A}_{i}\right)$ is an augmented ball space; hence also $(X, \mathcal{A})$ is an augmented ball space.

The definition of $\mathcal{A}$ yields that all $f_{i}$ as functions from $(X, \mathcal{A})$ to $\left(Y_{i}, \mathcal{A}_{i}\right)$ are ball continuous. We have to show that for any ball space $\left(Z, \mathcal{A}_{Z}\right)$ and function $g: Z \rightarrow X$ we have that $g:\left(Z, \mathcal{A}_{Z}\right) \rightarrow(X, \mathcal{A})$ is ball continuous if and only if for all $i \in I, f_{i} \circ g:\left(Z, \mathcal{A}_{Z}\right) \rightarrow\left(Y_{i}, \mathcal{A}_{i}\right)$ is ball continuous.

If $g$ is ball continuous, then by Lemma 4 and our remark at the start of this section, so are all $f_{i} \circ g$. For the converse, assume that all $f_{i} \circ g$ are ball continuous. Take $A \in \mathcal{A}$. Then by definition, there is some $i \in I$ and some $A_{i} \in \mathcal{A}_{i}$ such that $A=f_{i}^{-1}\left(A_{i}\right)$. Since $f_{i} \circ g$ is ball continuous, $g^{-1}(A)=$ $g^{-1}\left(f_{i}^{-1}\left(A_{i}\right)\right)=\left(f_{i} \circ g\right)^{-1}\left(A_{i}\right) \in \mathcal{A}_{Z}$. This shows that $g$ is ball continuous.

(2) Every ball structure on $X$ is a subset of $\mathcal{P}(X)$ and so it is an element of $\mathcal{P}(\mathcal{P}(X))$.

(3) Every set with at most one element carries a unique augmented ball space structure. Indeed, if the set is empty, then this is $(\emptyset,\{\emptyset\})$. If $X$ is a singleton, then $(X,\{X\})$ is a ball space, so by definition, $(X,\{\emptyset, X\})$ is an augmented ball space; this is the only augmented ball space structure on $X$.

Note that the definition (9) used in the proof also yields an initial object in the category of all ball spaces where the morphisms are assumed to be the surjective ball continuous functions. But if one of the $f_{i}$ 's is not surjective, it can happen that there is a ball $B_{i} \in \mathcal{A}_{i}$ such that $B_{i} \cap f_{i}(X)=\emptyset$, so that $f_{i}^{-1}\left(B_{i}\right)=\emptyset$.

The definitions of product and coproduct can be taken over from the category of ball spaces. If we use the construction described in (5), with every $\mathcal{B}_{i}$ replaced by $\mathcal{A}_{i}$, to derive an augmented ball space $(X, \mathcal{A})$ from augmented ball spaces $\left(Y_{i}, \mathcal{A}_{i}\right)$, then $\mathcal{A}$ will now contain $\emptyset$ (we take $B_{i}=\emptyset \in \mathcal{A}_{i}$ for some $i \in I$ ) and $X$ (we take $B_{i}=Y_{i} \in \mathcal{A}_{i}$ for all $\left.i \in I\right)$.

Further, if we apply the construction described in (6) to derive an augmented ball space $(X, \mathcal{A})$ from augmented ball spaces $\left(Y_{i}, \mathcal{A}_{i}\right)$, then $\mathcal{A}$ will again contain $\emptyset$ (we take $B_{i}=\emptyset \in \mathcal{A}_{i}$ for all $i \in I$ ) and $X$ (we take $A_{i}=Y_{i} \in \mathcal{A}_{i}$ for all $i \in I$ ).

Observe that now also

$$
\mathcal{A}^{\prime}:=\left\{\iota_{i}\left(A_{i}\right) \mid i \in I, A_{i} \in \mathcal{A}_{i}\right\}
$$

renders all embeddings $\iota_{i}$ ball continuous since $\emptyset \in \mathcal{A}_{i}$. However, in all cases where $\mathcal{A}^{\prime}$ differs from the set $\mathcal{A}$ obtained from (6), examples can be constructed that show that this is not a coproduct.

Since the category of augmented ball spaces is topological, it must also admit final objects (cf. 9]). We can present them explicitly. Take augmented ball spaces $\left(Y_{i}, \mathcal{A}_{i}\right)$, a set $X$, and functions $f_{i}: Y_{i} \rightarrow X, i \in I$. We set

$$
\mathcal{A}:=\left\{A \subseteq X \mid \forall i \in I: f_{i}^{-1}(A) \in \mathcal{A}_{i}\right\} .
$$


Then all $f_{i}$ as functions from $\left(Y_{i}, \mathcal{A}_{i}\right)$ to $(X, \mathcal{A})$ are ball continuous. Further, $\emptyset \in \mathcal{A}$ since $f_{i}^{-1}(\emptyset)=\emptyset \in \mathcal{A}_{i}$ for all $i \in I$, and $X \in \mathcal{A}$ since $f_{i}^{-1}(X)=Y_{i} \in \mathcal{A}_{i}$ for all $i \in I$. It remains to show that for any ball space $\left(Z, \mathcal{A}_{Z}\right)$ and function $g: X \rightarrow Z$ we have that $g:(X, \mathcal{A}) \rightarrow\left(Z, \mathcal{A}_{Z}\right)$ is ball continuous if and only if for all $i \in I$, $g \circ f_{i}:\left(Y_{i}, \mathcal{A}_{i}\right) \rightarrow\left(Z, \mathcal{A}_{Z}\right)$ is ball continuous.

If $g$ is ball continuous, then by Lemma 4 and our remark at the start of this section, so are all $g \circ f_{i}$. For the converse, assume that all $g \circ f_{i}$ are ball continuous and take $A \in \mathcal{A}_{Z}$. Then $f_{i}^{-1}\left(g^{-1}(A)\right)=\left(g \circ f_{i}\right)^{-1}(A) \in \mathcal{A}_{i}$ for all $i \in I$ by continuity. Hence by $10, g^{-1}(A) \in \mathcal{A}$, showing that $g$ is ball continuous.

We observe that this definition does not work in the category of ball spaces. For example, consider ball spaces $\left(X,\left\{B_{1}\right\}\right)$ and $\left(X,\left\{B_{2}\right\}\right)$ with $B_{1} \neq B_{2}$ and $f_{1}$ and $f_{2}$ the identity function of $X$. Then there is no subset of $X$ with preimage $B_{1}$ under $f_{1}$ and $B_{2}$ under $f_{2}$, so 10 renders the empty set when $\emptyset$ and the whole set are not balls.

\section{Hybrid Ball Spaces}

As a final topic in this article we investigate the following question:

Given two spherically complete ball spaces $\left(X, \mathcal{B}_{1}\right)$ and $\left(X, \mathcal{B}_{2}\right)$ on the same set $X$, which operations of forming new balls from the balls in $\mathcal{B}_{1} \cup \mathcal{B}_{2}$ will preserve spherical completeness?

A first step is provided by the following proposition; the easy proof is left to the reader.

Proposition 11. If $\left(X, \mathcal{B}_{1}\right)$ and $\left(X, \mathcal{B}_{2}\right)$ are $\mathbf{S}_{1}$ ball spaces, then so is the ball space $\left(X, \mathcal{B}_{1} \cup \mathcal{B}_{2}\right)$. The same holds with $\mathbf{S}_{2}$ or $\mathbf{S}_{5}$ in place of $\mathbf{S}_{1}$.

Note that the assertion may become false if we replace $\mathbf{S}_{1}$ by $\mathbf{S}_{3}$ or $\mathbf{S}_{4}$. Indeed, the intersection of a nest in $\mathcal{B}_{1}$ may properly contain a largest (or maximal) ball which does not remain the largest (or a maximal) ball contained in the intersection in $\mathcal{B}_{1} \cup \mathcal{B}_{2}$.

Having obtained $\mathcal{B}=\mathcal{B}_{1} \cup \mathcal{B}_{2}$, the next question is how to create new balls from the balls in $\mathcal{B}$ without losing spherical completeness. The results of taking unions and intersections are discussed in [1]. In the next section, we present a particular case.

\subsection{Closure under finite unions of balls.}

Take a ball space $(X, \mathcal{B})$. By $\mathrm{f}-\mathrm{un}(\mathcal{B})$ we denote the set of all unions of finitely many balls in $\mathcal{B}$. In $[\mathbf{1}$, the following theorem is proven:

Theorem 12. If $(X, \mathcal{B})$ is an $\mathbf{S}_{1}^{c}$ ball space, then so is $(X, \mathrm{f}-\mathrm{un}(\mathcal{B}))$.

For the convenience of the reader, we repeat the proof here. We need a lemma.

Lemma 13. If $\mathcal{S}$ is a maximal centered system of balls in $\mathrm{f}-\mathrm{un}(\mathcal{B})$ (that is, no subset of $\mathrm{f}-\mathrm{un}(\mathcal{B})$ properly containing $\mathcal{S}$ is a centered system), then there is a subset $\mathcal{S}_{0}$ of $\mathcal{S}$ which is a centered system in $\mathcal{B}$ and has the same intersection as $\mathcal{S}$.

Proof. It suffices to prove the following: if $B_{1}, \ldots, B_{n} \in \mathcal{B}$ such that $B_{1} \cup \ldots \cup B_{n} \in$ $\mathcal{S}$, then there is some $i \in\{1, \ldots, n\}$ such that $B_{i} \in \mathcal{S}$. 
Suppose that $B_{1}, \ldots, B_{n} \in \mathcal{B} \backslash \mathcal{S}$. By the maximality of $\mathcal{S}$ this implies that for each $i \in\{1, \ldots, n\}, \mathcal{S} \cup\left\{B_{i}\right\}$ is not centered. This in turn means that there is a finite subset $\mathcal{S}_{i}$ of $\mathcal{S}$ such that $\cap \mathcal{S}_{i} \cap B_{i}=\emptyset$. But then $\mathcal{S}_{1} \cup \ldots \cup \mathcal{S}_{n}$ is a finite subset of $\mathcal{S}$ such that

$$
\bigcap\left(\mathcal{S}_{1} \cup \ldots \cup \mathcal{S}_{n}\right) \cap\left(B_{1} \cup \ldots \cup B_{n}\right)=\emptyset .
$$

This yields that $B_{1} \cup \ldots \cup B_{n} \notin \mathcal{S}$, which proves our assertion.

Proof of Theorem 12. Take a centered system $\mathcal{S}^{\prime}$ of balls in f-un $(\mathcal{B})$. Centered systems of balls in a ball space are inductively ordered by inclusion. Hence there is a maximal centered system $\mathcal{S}$ of balls in f-un $(\mathcal{B})$ which contains $\mathcal{S}^{\prime}$. By Lemma 13 there is a centered system $\mathcal{S}_{0}$ of balls in $\mathcal{B}$ such that $\bigcap \mathcal{S}_{0}=\bigcap \mathcal{S} \subseteq \bigcap \mathcal{S}^{\prime}$. Since $(X, \mathcal{B})$ is an $S_{1}^{c}$ ball space, we have that $\bigcap \mathcal{S}_{0} \neq \emptyset$, which yields that $\bigcap \mathcal{S}^{\prime} \neq \emptyset$. This proves that $(X, \mathrm{f}-\mathrm{un}(\mathcal{B}))$ is an $\mathrm{S}_{1}^{c}$ ball space.

This theorem becomes false if " $\mathbf{S}_{1}^{c}$ " is replaced by " $\mathbf{S}_{1}$ ".

Example 14. For every $i \in \mathbb{N}$ we let $p_{i}$ be the $i$-th prime. For every $i \in \mathbb{N}$ we define a ball $B_{i} \subset \mathbb{R}$ by

$$
B_{i}:=\left(0, \frac{1}{p_{i}}\right) \backslash\left\{\frac{1}{p_{i}^{j}} \mid j \in \mathbb{N} \text { with } p_{i}^{j}>p_{i+1}\right\},
$$

and set $\mathcal{B}:=\left\{B_{i} \mid i \in \mathbb{N}\right\}$. Then for $i \neq j, B_{i}$ and $B_{j}$ are not comparable by inclusion. Therefore, $\mathcal{B}$ admits no nests with more than one ball and is thus spherically complete. But

$$
B_{i} \cup B_{i+1}=\left(0, \frac{1}{p_{i}}\right)
$$

since all the real numbers in $\left(0, \frac{1}{p_{i}}\right)$ that are missing in $B_{i}$ are elements of $B_{i+1}$. Further, $\left(B_{i} \cup B_{i+1}\right)_{i \in \mathbb{N}}$ is a nest in $(\mathbb{R}, \mathrm{f}-\operatorname{un}(\mathcal{B}))$. As it has empty intersection, $(\mathbb{R}, \mathrm{f}-\mathrm{un}(\mathcal{B}))$ is not spherically complete.

This leads us to the following question:

Under which other conditions than $\mathbf{S}_{1}^{c}$ is spherical completeness preserved under taking finite unions?

We discuss one special case in the next section, starting with a ball space that is not $\mathbf{S}_{1}^{c}$.

6.2. Bar-bells. As already mentioned in the Introduction, a natural example of algebraic structures on which two distinct ball spaces appear naturally are ordered abelian groups and ordered fields. On the one hand such a structure $(G, \leq)$ admits a natural valuation which is nontrivial if the ordering is nonarchimedean (cf. Section 2.3p. This gives rise to an ultrametric space, from which in turn we can derive the ball space $\left(G, \mathcal{B}_{u}\right)$ where $\mathcal{B}_{u}$ consists of all closed ultrametric balls (cf. Section 2.2. . On the other hand, one can consider the order ball space $\left(G, \mathcal{B}_{o}\right)$ where $\mathcal{B}_{o}$ consists of all nonempty closed bounded intervals. In $[\mathbf{8}$ it is shown that if $(G, \leq)$ is symmetrically complete, then $\left(G, \mathcal{B}_{o}\right)$ is spherically complete, and that if $\left(G, \mathcal{B}_{o}\right)$ is spherically complete, then so is $\left(G, \mathcal{B}_{u}\right)$. Hence if $(G, \leq)$ is symmetrically complete, then $(G, \mathcal{B})$ is spherically complete for $\mathcal{B}=\mathcal{B}_{u} \cup \mathcal{B}_{o}$ by Proposition 11 . 
While $\left(G, \mathcal{B}_{u}\right)$ is always an $\mathbf{S}_{2}^{c}$ ball space once it is spherically complete (cf. [1]), $\left(G, \mathcal{B}_{o}\right)$ and $(G, \mathcal{B})$ are $\mathbf{S}_{1}^{c}$ if and only if $G=\mathbb{R}$ (with the canonical ordering). Hence if $G \neq \mathbb{R}$, we cannot apply Theorem 12 here.

A bar-bell is a subset of $G$ obtained from a nonempty closed bounded interval $[a, b]$ by joining it with ultrametric balls centered in $a$ and in $b$; it can thus be written as $B_{\alpha}(a) \cup[a, b] \cup B_{\beta}(b)$ with $\alpha, \beta \in u G$ and $a \leq b$.

Lemma 15. For every symmetrically complete ordered abelian group or field, the ball space of bar-bells is spherically complete.

Proof. Take a nest $\mathcal{N}$ of bar-bells. W.l.o.g. we may assume that $\mathcal{N}=\left(B_{i}\right)_{i<\kappa}$ where $\kappa$ is the cofinality of the nest and that $i<j<\kappa \Rightarrow B_{j} \subsetneq B_{i}$. We write

$$
B_{i}=B_{\alpha_{i}}\left(a_{i}\right) \cup\left[a_{i}, b_{i}\right] \cup B_{\beta_{i}}\left(b_{i}\right)
$$

with $\alpha_{i}, \beta_{i} \in u G$ and $a_{i} \leq b_{i}$.

If there is a nest of ultrametric balls that has an intersection which is a subset of the intersection of $\mathcal{N}$, then we are done. Hence assume that there is no such nest. We will construct a sequence $\left(i_{\nu}\right)_{\nu<\kappa}$ that is cofinal in $\kappa$, such that

$$
B_{i_{\nu+1}} \subsetneq\left[a_{i_{\nu}}, b_{i_{\nu}}\right] \subseteq B_{i_{\nu}} .
$$

Then

$$
\bigcap_{i<\kappa} B_{i}=\bigcap_{\nu<\kappa} B_{i_{\nu}}=\bigcap_{\nu<\kappa}\left[a_{i_{\nu}}, b_{i_{\nu}}\right]
$$

and we are done again.

We take $i_{0}=0$. Assume that for some $\nu<\kappa$ we have already chosen $i_{\mu}$ for all $\mu \leq \nu$ such that (11) holds with $\mu$ in place of $\nu$. By our assumption there must be some $i_{\nu+1}<\kappa, i_{\nu+1}>i_{\nu}$, such that $B_{\alpha_{i_{\nu+1}}}\left(a_{i_{\nu+1}}\right) \not \subset B_{\alpha_{i_{\nu}}}\left(a_{i_{\nu}}\right)$ and $B_{\beta_{i_{\nu+1}}}\left(b_{i_{\nu+1}}\right) \not \subset$ $B_{\beta_{i_{\nu}}}\left(b_{i_{\nu}}\right)$. Then $B_{\alpha_{i_{\nu+1}}}\left(a_{i_{\nu+1}}\right) \cap B_{\alpha_{i_{\nu}}}\left(a_{i_{\nu}}\right)=\emptyset$ and $B_{\beta_{i_{\nu+1}}}\left(b_{i_{\nu+1}}\right) \cap B_{\beta_{i_{\nu}}}\left(b_{i_{\nu}}\right)=\emptyset$. Since $B_{i_{\nu+1}} \subset B_{i_{\nu}}$, we must have that $a_{i_{\nu}}<B_{i_{\nu+1}}<b_{i_{\nu}}$. Therefore, (11) holds.

Now assume that $\lambda<\kappa$ is a limit ordinal and that we have already chosen $i_{\nu}$ and constructed $B_{i_{\nu}}^{\prime}$ for all $\nu<\lambda$ such that 11 holds. Then we choose any $i \in I$ that is larger than all $i_{\nu}$ (which exists since $\lambda$ is smaller than the cofinality $\kappa$ ) and set $i_{\lambda}:=i$ and proceed as above with $\nu=\lambda$.

Any finite union $S$ of closed bounded intervals and ultrametric balls that is convex is a bar-bell. This is seen as follows. Suppose that the union of the intervals $\left[a_{i}, b_{i}\right], 1 \leq i \leq m$, and the balls $B_{\alpha_{j}}\left(c_{j}\right), 1 \leq j \leq n$, is convex. Since ultrametric balls with equal centers are comparable by inclusion, by listing only the largest ones we may assume that all $c_{j}$ are distinct. Further, we can add the singleton ball $B_{\infty}\left(a_{i}\right)$ or $B_{\infty}\left(b_{i}\right)$ to the balls $B_{\alpha_{j}}\left(c_{j}\right)$ in case $a_{i}$, or $b_{i}$ respectively, is not contained in any of the balls. Let $\min _{1 \leq j \leq n} c_{j}=c_{j_{1}}$ and $\max _{1 \leq j \leq n} c_{j}=c_{j_{2}}$. Then $S=B_{\alpha_{j_{1}}}\left(c_{j_{1}}\right) \cup\left[c_{j_{1}}, c_{j_{2}}\right] \cup B_{\alpha_{j_{2}}}\left(c_{j_{2}}\right)$.

From this fact together with Lemma 15 we obtain Theorem 3

Let us collect a few special properties of the ball space $\mathcal{B}$ of convex finite unions of closed bounded intervals and ultrametric balls, which could be helpful in answering the question stated after Theorem 3 .

1) All of its balls can be expressed by a union of at most 3 balls from the two generating ball spaces $\mathcal{B}_{u}$ and $\mathcal{B}_{o}$. 
2) Every ball in $\mathcal{B}_{u} \cap \mathcal{B}_{o}$ is a singleton.

3) Both $\mathcal{B}_{u}$ and $\mathcal{B}_{o}$ are closed under finite nonempty intersections.

4) If $\mathcal{N}$ is a nest in $\mathcal{B}$, then there is a nest $\mathcal{N}^{\prime}$ in $\mathcal{B}_{u}$ or in $\mathcal{B}_{o}$ such that $\bigcap \mathcal{N}^{\prime} \subseteq \bigcap \mathcal{N}$.

An attempt to generalize the notion of convexity to arbitrary ball spaces could be to call a finite collection of balls pseudo convex if it is of the form $\left\{B_{1}, \ldots, B_{n}\right\}$ with $B_{i} \cap B_{i+1} \neq \emptyset$ for $1 \leq i<n$.

Open question: Does the closure of a ball space under unions of finite pseudo convex collections of balls always preserve spherical completeness, even if the ball space fails to be $\mathbf{S}_{1}^{c}$ ? If not, what other conditions could ensure this?

\section{References}

[1] H. Ćmiel, F.-V. Kuhlmann and K. Kuhlmann, A generic approach to measuring the strength of completeness/compactness of various types of spaces and ordered structures, to appear in Revista de la Real Academia de Ciencias Exactas, Físicas y Naturales. Serie A. Matemáticas, arXiv:1905.09930v2

[2] W. Kubis and F.-V. Kuhlmann, Chain intersection closures, Topology Appl., 262 (2019), 11-19, arXiv:1810.05832v1.

[3] F.-V. Kuhlmann, Maps on ultrametric spaces, Hensel's lemma, and differential equations over valued fields, Commun. Algebra 39 (5) (2011), 1730-1776.

[4] F.-V. Kuhlmann, K. Kuhlmann and M. Paulsen, The Caristi-Kirk fixed point theorem from the point of view of ball spaces, J. Fixed Point Theory Appl. 20 (3) (2018), Paper No. 107.

[5] F.-V. Kuhlmann, K. Kuhlmann and F. Sonaallah, Coincidence point theorems for ball spaces and their applications, Ordered algebraic structures and related topics, Luminy, Contemporary Mathematics 697, 211-226, American Mathematical Society, Providence, 2017.

[6] K. Kuhlmann and F.-V. Kuhlmann, A common generalization of metric and ultrametric fixed point theorems, Forum Math. 27 (1) (2015), 303-327; and: Correction to "A common generalization of metric, ultrametric and topological fixed point theorems", Forum Math. 27 (1) (2015), 329-330; alternative corrected version available at: http://math.usask.ca/fvk/GENFPTAL.pdf

[7] K. Kuhlmann and F.-V. Kuhlmann, Fixed point theorems for spaces with a transitive relation, Fixed Point Theory 18 (2) (2017), 663-672.

[8] K. Kuhlmann, F.-V. Kuhlmann and S. Shelah, Symmetrically complete ordered sets, abelian groups and fields, Isr. J. Math. 208 (2015), 261-290.

[9] G. Preuss, Foundations of Topology: an Approach to Convenient Topology, Kluwer Academic Publishers, Dordrecht, 2002.

[10] S. Prieß-Crampe and P. Ribenboim, Generalized ultrametric spaces. I, Abh. Math. Semin. Univ. Hamb. 66 (1996), 55-73.

[11] S. Prieß-Crampe and P. Ribenboim, The common point theorem for ultrametric Spaces, Geom. Dedicata 72 (1) (1998), 105-110.

[12] S. Prieß-Crampe and P. Ribenboim, Fixed point and attractor theorems for ultrametric spaces, Forum Math. 12 (1) (2000), 53-64.

[13] F. Schwarz, Description of the topological universe hull, in H. Ehrig et al., editors, Categorical Methods in Computer Science With Aspects from Topology, Lecture Notes in Computer Science 393, Springer, Berlin, 1989, 325-332. 
[14] S. Shelah, Quite complete real closed fields, Isr. J. Math. 142 (2004), 261-272.

René Bartsch

A.-v.-Humboldt-Gymnasium, Institute of Mathematics, Makarenkostr. 54,

17491 Greifswald,

Germany

bartsch@humboldtgreifswald.de

math@marvinius.net
Katarzyna Kuhlmann

ul. Wielkopolska 15 ,

70-451 Szczecin,

Franz-Viktor Kuhlmann,

Institute of Mathematics,

ul. Wielkopolska 15 ,

70-451 Szczecin,

Poland

Katarzyna.Kuhlmann@usz.edu.pl fvk@usz.edu.pl 